\title{
Refining practice: TID metrics for CZT systems
}

\author{
Eliana Reyes, $\mathrm{MD}, \mathrm{PhD}^{\mathrm{a}, \mathrm{b}}$ \\ a The PET Imaging Centre, St Thomas' Hospital, King's College London, London, United Kingdom \\ b Royal Brompton and Harefield NHS Foundation Trust, London, United Kingdom
}

Received Jun 5, 2019; accepted Jun 5, 2019

doi: $10.1007 / \mathrm{s} 12350-019-01801-0$

\section{See related article, pp. 1180-1189}

Systematic collection of data to improve health care had in Florence Nightingale (1820-1910) one of its most tenacious advocates. She was not only the founder of modern nursing and the most regarded nurse in history but also one of the pioneers in the use of evidence to inform clinical practice. Fast forward to the 21st century, advancing technology is summoning an evergrowing number of advocates of big and better data collection to improve our understanding of the natural history of diseases, and how the physical and social environment impacts on health. In the field of cardiovascular medicine, analysis of (large-scale) data has proved highly valuable in the evaluation of the efficacy of contemporary diagnostic imaging and its many markers of disease.

Highly valued in daily nuclear cardiology practice but often overlooked in the design of research studies, transient ischaemic dilatation (TID) is a well-recognized non-perfusion marker of both clinically and prognostically significant obstructive coronary artery disease (CAD) on radionuclide myocardial perfusion imaging (MPI). ${ }^{1}$ It has been nearly 40 years since TID was first described in the literature as the appearance of a larger left ventricular (LV) cavity on the stress images compared to rest. $^{2}$ Since, this phenomenon has been attributed to (a) stress-induced LV contractile dysfunction or stunning with increased LV volumes, and (b) extensive or global subendocardial inducible

Reprint requests: Eliana Reyes, MD, PhD, Royal Brompton and Harefield NHS Foundation Trust, London, United Kingdom; e.reyes@rbht.nhs.uk

J Nucl Cardiol 2020;27:1190-2.

$1071-3581 / \$ 34.00$

Copyright (c) 2019 American Society of Nuclear Cardiology. hypoperfusion resulting in an apparent increase in LV cavity size with stress. Both proposed mechanisms are supported by evidence, and it is probable that the prevalence of one mechanism over the other would vary depending on the characteristics of the patient population studied; possibly also on the modality of stress employed. ${ }^{3,4}$ In my experience, visually obvious "barn door' TID, which sometimes is associated with a distinctive change in geometry with the LV cavity appearing slightly more "rounded" or "curved" on the stress images than at rest, does not require further verification by quantification. Frequently, this relative increase in LV cavity size with stress appears rather subtle. In such case, a quantitative measurement of TID, expressed as the ratio between stress and rest LV volumes, aids visual interpretation. In this context, TID is one of the most useful metrics one can rely on. In this issue of the Journal, the work of $\mathrm{Hu}$ et al aimed at establishing the upper reference value of TID measurements for the new generation of cadmium-zinc-telluride (CZT) gamma cameras. ${ }^{5}$ The researchers hypothesized that this value might be different not only from those already reported for conventional gamma cameras but also between the two commercially available CZT systems. By sourcing input from a vast international multicenter registry, REFINE SPECT (registry of fast myocardial perfusion imaging with next generation SPECT), the researchers had many cases at hand, enabling them to evaluate the suitability of a large number of observations to answer the research question. Data were meticulously curated, and cases carefully selected to assemble a cohort with a low probability for obstructive CAD from which normality could be estimated. The researchers also generated camera-, stress protocol-, and tracer-specific TID limits, which was subject to one condition: having sufficient observations in each sub-group for meaningful results.

The findings of this work confirm the results from previous studies; the upper boundary of normal stress/ rest LV volume ratios calculated from summed (nonECG-gated) tomograms is influenced by several factors. 
Quantitative TID limits (97.5th percentile) varied from 1.18 to 1.52 across the study sub-groups. CZT gammacamera system vendor, however, does not appear to be one of those modifying factors with cardiac-dedicated D-SPECT and GE Discovery showing similar upper TID limits for exercise Tc-99m sestamibi SPECT protocol (1.20 vs 1.22). Throughout the study, it becomes clear that quoting a single TID limit value as reference for all SPECT MPI studies without consideration of image processing, stress or tracer protocol may fall short of one's ideal of best practice. It has already been described in the literature that TID measurements vary according to the application used for automatic segmentation, analysis, and image display and hence results from the several software packages currently available (e.g., 4DM, QPS/QGS, Emory toolbox, among others) may not be interchangeable. Moreover, as demonstrated by $\mathrm{Hu}$ et al for the new generation of gamma cameras, it has already been suggested that TID values derived from images obtained with conventional SPECT systems can be affected by the modality of stress, type of tracer, and protocol used. ${ }^{1}$

Although the need for multiple TID thresholds goes against the pursuit of simplicity, let's not forget that quantitative TID is most useful in cases in which changes in LV cavity size from stress to rest appear equivocal or borderline to the naked eye. In this context, the final interpretation of the images will require consideration not only of quantitative TID but also of clinical history, physical examination, prior investigations, and stress testing results to determine, on an individual basis, whether the apparent increase in LV cavity size with stress is clinically and prognostically relevant. An important observation from the study is that stress/rest LV volume data were not normally distributed, indicating that a non-parametric method should be employed for reporting upper limits of reference for stress/rest LV volumes ratios. The absence of a normal distribution may deter us from extrapolating the results to healthy, "normal" individuals. The assumption held here is that the study cohort is representative of the patients we see in our daily clinics and therefore the study findings are valid for the purpose at hand. The use of a large data source facilitated the exclusion of patients at risk of obstructive CAD while maintaining sufficient number of observations for comparison. However, it was not feasible to exclude patients at risk of non-coronary hypoperfusion. Hypertensive heart disease may result in an apparent increase in LV cavity size with stress. However, hypertension is highly prevalent and controlling for this variable would have made the study unattainable. The message here is that when uncertain about TID, one must consider the multiple factors that may influence this metric and should be wary about using a single value to base one's judgement on.

There was no direct comparison with conventional gamma-camera systems nor were data on TID limits for upright or prone acquisitions. Regarding the former, it would not be wise to extrapolate values from supine, simply because postural changes may impact on ventricular volumes, ${ }^{6}$ although one cannot be certain about the extent of such effect when using ratios rather than absolute values. Finally, the study was not intended to explain the observed variability in TID limits. One must consider that multiple mechanisms may come into play; physiological, for example, the cardiovascular response to exercise vs vasodilator stress can have a differential effect on LV contractility and volumes; and practical, for instance, tracer injection-to-image acquisition time can impact on LV volumes after cardiac stress. Studies like the one conducted by $\mathrm{Hu}$ et al reinforce the value of large registries in clinical research to address issues that may affect our practice; one must continue cultivating the habit of institutional and multi-national collaboration, and continue supporting systematic data collection to help inform practice and generate hypothesis. Data is becoming increasingly powerful; let's ensure that it is collected and analyzed to the highest possible standards. Florence Nightingale would be proud.

\section{Disclosure}

No conflict of interest to declare.

\section{References}

1. Alama M, Labos C, Emery H, Iwanochko RM, Freeman M, Husain $\mathrm{M}$, et al. Diagnostic and prognostic significance of transient ischemic dilation (TID) in myocardial perfusion imaging: A systematic review and meta-analysis. J Nucl Cardiol 2018;25:72437.

2. Stolzenberg J. Dilatation of the left ventricular cavity on stress thallium scan as an indicator of ischemic disease. Clin Nucl Med 1980;5:289-91

3. Emmett L, Ng A, Ha L, Russo R, Mansberg R, Zhao W, et al. Comparative assessment of rest and post-stress left ventricular volumes and left ventricular ejection fraction on gated myocardial perfusion imaging (MPI) and echocardiography in patients with transient ischaemic dilation on adenosine MPI: Myocardial stunning or subendocardial hypoperfusion? J Nucl Cardiol 2012;19:735-42.

4. Mohammad R, Hojjati MR, Muthupillai R, Wilson JM, Preventza OA, Cheong BYC. Assessment of perfusion and wall-motion abnormalities and transient ischemic dilation in regadenoson stress 
cardiac magnetic resonance perfusion imaging. Int $\mathrm{J}$ Cardiovasc Imaging 2014;30:949-57.

5. Hu LH, Sharir T, Miller R, Einstein A, Fish M, Ruddy T, et al. Upper reference limits of transient ischemic dilation for different protocols on new-generation cadmium zinc telluride cameras: A report from REFINE SPECT registry. J Nucl Cardiol 2019. https://d oi.org/10.1007/s12350-019-01730-y.
6. Poliner LR, Dehmer GJ, Lewis SE, Parkey RW, Blomqvist CG, Willerson JT. Left ventricular performance in normal subjects: A comparison of the responses to exercise un the upright and supine positions. Circulation 1980;62:528-34.

Publisher's Note Springer Nature remains neutral with regard to jurisdictional claims in published maps and institutional affiliations. 